\title{
Cadmium Telluride X-ray pad detectors with different
} passivation dielectrics

\section{Gädda, Akiko}

2019-04-21

Gädda , A , Ott , J , Karadzhinova-Ferrer , A , Golovleva , M , Kalliokoski , M , Winkler , A , Luukka , P \& Härkönen , J 2019 , ' Cadmium Telluride X-ray pad detectors with different passivation dielectrics ' , Nuclear Instruments \& Methods in Physics Research. Section A: Accelerators, Spectrometers, Detectors, and Associated Equipment , vol. 924 , pp. 33-37 . https://doi.org/10.1016/j.n

http://hdl.handle.net/10138/328224

https://doi.org/10.1016/j.nima.2018.08.063

cc_by_nc_nd

acceptedVersion

Downloaded from Helda, University of Helsinki institutional repository.

This is an electronic reprint of the original article.

This reprint may differ from the original in pagination and typographic detail.

Please cite the original version. 


\title{
Cadmium Telluride X-ray pad detectors with different passivation dielectrics
}

\author{
A. Gädda ${ }^{\mathrm{a},}$, J. Ott $^{\mathrm{a}}$, A. Karadzhinova-Ferrer ${ }^{\mathrm{b}}$, M. Golovleva $^{\mathrm{a}, \mathrm{c}}$, M. Kalliokoski $^{\mathrm{b}}$, \\ A. Winkler ${ }^{\mathrm{a}, \mathrm{d}}$, P. Luukka ${ }^{\mathrm{a}}$, J. Härkönen ${ }^{\mathrm{b}}$ \\ ${ }^{a}$ Helsinki Institute of Physics, Gustaf Hällströmin katu 2, 00014 University of Helsinki, Finland \\ ${ }^{b}$ Ruder Bošković Institute, Bijenička cesta 54, 10000 Zagreb, Croatia \\ ${ }^{c}$ Lappeenranta University of Technology, Skinnarilankatu 34, 53850 Lappeenranta, Finland \\ ${ }^{d}$ Detection Technology Plc, Ahventie 4B, FI-02170 Espoo, Finland
}

\begin{abstract}
The suitability of two low-temperature dielectric passivation layer processes for the fabrication of Cadmium Telluride ( $\mathrm{CdTe}$ ) X-ray detectors has been investigated. The CdTe crystals with a size of $(10 \times 10 \times 1) \mathrm{mm}^{3}$ were coated with sputtered aluminum nitride $(\mathrm{AlN})$ or with aluminum oxide $\left(\mathrm{Al}_{2} \mathrm{O}_{3}\right)$ grown by the atomic layer deposition (ALD) method. The metallization contacts of the detectors were made by titanium tungsten (TiW) and gold $(\mathrm{Au})$ metal sputtering depositions. The pad detector structures were patterned with proximity-contactless photolithography techniques followed by lift-off patterning of the electrodes. The detector properties were characterized at room temperature by Transient Current Technique (TCT) measurements. The obtained results were compared and verified by numerical TCAD simulations of the detector response. Our results indicate that higher signal charge was collected from samples with $\mathrm{Al}_{2} \mathrm{O}_{3}$. Furthermore, no significant laser light induced signal decay by CdTe material polarization was observed within order of 30 minutes of continuous illumination.

Keywords: Cadmium Telluride (CdTe), Atomic Layer Deposition (ALD), X-ray detector
\end{abstract}

Email address: akiko.gadda@helsinki.fi (A. Gädda) 


\section{Introduction}

Photon detectors made of high atomic number $(\mathrm{Z})$ semiconductor materials are utilized for a wide variety of applications, such as for spectroscopy of nuclear isotopes, or medical imaging [1,2]. Generally desired properties of such detector systems are good energy resolution $(\Delta \mathrm{E} / \mathrm{E})$ and an X-ray image quality that is as sharp as possible. One of the semiconductor materials that frequently used for room temperature spectroscopic applications is CdTe [3, 4]. Its high effective atomic number $\mathrm{Z}_{\text {eff }}=50$ is essential for the good attenuation of up to several hundred $\mathrm{keV}$ of ionizing radiation, and the band gap of $1.44 \mathrm{eV}$ allows low noise operation at room temperature.

Assuming an appropriate signal generation in a CdTe detector of certain thickness, a measure of spectroscopic or imaging performance is the Charge Collection Efficiency (CCE) [5, 6]. The CCE is simply the ratio of electrical charge collected by electrodes divided by the amount of deposited charge. The charge transport in a semiconductor detector and CCE are often modelled by the well-known Hecht equation published in 1932 [7]. The Hecht equation implies that, in addition to geometrical parameters, charge transport in the electric field depends on the product of carrier mobility and trapping lifetime. Qualitatively, good CCE results in improved energy resolution and thus better image quality. The CCE is reduced by trapping/ recombination of photon generated charge carriers. Trapping/ recombination processes take place inside of the active volume of a detector as well as at the front and back surfaces [8]. If the ratio of detector thickness (L) and drift velocity of charge carriers $\left(v_{\text {drift }}\right)$ is larger than the charge lifetime $\left(\tau_{e, h}\right)$, then the CCE will be degraded. The $\tau_{e, h}$ is inversely proportional to the concentration of the trapping centers, i.e defects in the bulk of semiconductor. Due to the complex growth process of CdTe [9], the concentration of bulk defects (i.e. Te inclusion) is almost always very high. Typical values are around $3 \times 10^{6}$ inclusion $/ \mathrm{cm}^{3}[10]$.

The carrier drift velocity is directly proportional to the product of the carrier mobility $\left(\mu_{e, h}\right)$ and the local electric field $(\mathbf{E}(\mathbf{x}))$; however, saturation effects occur in CdTe at the field strength of $>100 \mathrm{~V} / \mathrm{cm}$ for electrons $\left(\tau_{e} \approx 5-10 \mu \mathrm{s}\right)[11,9]$ and several $\mathrm{kV} / \mathrm{cm}$ for holes, due to the short $\tau_{h} \leq 1 \mu \mathrm{s}$ of holes. Thus, high voltage operation of a CdTe detector is beneficial in order to reach saturation drift velocity of electrons. The electron mobility $\left(\mu_{e}\right)$ in CdTe is in the order of $1100 \mathrm{~cm}^{2} / \mathrm{Vs}$, which is comparable with electron mobility e.g. in silicon [12]. The hole mobility $\left(\mu_{h}\right)$ in CdTe is in turn about an order of magnitude less than $\left(88 \mathrm{~cm}^{2} / \mathrm{Vs}\right)$ [12]. This suggests that it is more favorable to collect a signal which is dominantly formed by electrons, since most of the holes are lost due to trapping in CdTe bulk [5, 13]. Moreover, poor transport properties of holes in CdTe are known to cause a "hole tailing" effect, 
which results in an asymmetric broadening of peaks in measured spectra $[6,14]$.

In order to minimize signal losses due to surface recombination processes, a proper field insulation layer on the CdTe detector surfaces is needed for the formation of the CdTe Schottky diode. This can be achieved by implementing a dielectric thin film on the surface. Dielectric thin films often have certain electrical charge, which is a complex combination of e.g. interface charge, mobile ionic charge and fixed oxide charge [8]. If the oxide charge is positive, then the Coulomb force is repulsing holes from the damaged surface, thus providing electrical passivation of hole current. In case of negative oxide charge, a similar field effect passivation is established for electrons. The electrical passivation is also needed in order to provide resistive insulation between the electrodes of a segmented detector, and furthermore, provide protection against environmental effects such as moisture, corrosion, mechanical damages, or ambient light that would induce additional noise.

The electrical passivation of CdTe by deposition of dielectric thin films is challenging due to thermal expansion properties of CdTe crystals, which limits the maximum processing temperature to about $150^{\circ} \mathrm{C}[15,16]$. It is also well-known that the electrical and mechanical quality of dielectric CVD films typically improve with respect to the increasing deposition temperature [17]. In this report two thin film passivation materials, aluminum nitride (AlN) and aluminum oxide $\left(\mathrm{Al}_{2} \mathrm{O}_{3}\right)$, were studied. They were both deposited at a low temperature by using magnetron sputtering and Atomic Layer Deposition (ALD), respectively. The applied ALD method is based on the successive, separated, and self-terminating gas-solid reactions of typically two gaseous precursors and the deposition may take place at low temperature, compatible with CdTe detector processing $[18,19]$. Moreover, studies performed on silicon solar cells $[20,21]$ and particle detectors $[22,23]$ indicate that $\mathrm{Al}_{2} \mathrm{O}_{3}$ has a negative oxide charge, thus providing field effect passivation for electrons and allowing preferred signal formation mode for CdTe photon detectors. In this report, the passivation effects have been studied by Transient Current Technique (TCT) by recording current transients from laser illuminated CdTe pad detectors.

\section{Design and Processing}

The starting material is detector grade $\left(>10^{9} \Omega \cdot \mathrm{cm}\right.$ bulk resistivity) and (111) oriented crystal dies that were obtained from Acrorad Ltd. [24]. Crystals sizes are $(10 \times 10) \mathrm{mm}^{2}$ and $1 \mathrm{~mm}$ in thickness. As shown in Figure 1a, the chip layout contains a $(5.5 \times 5.5) \mathrm{mm}^{2}$ pad detector at the middle of the $1 \mathrm{~cm}^{2}$ crystal front plane. It is surrounded by a single $200 \mu \mathrm{m}$ wide guard ring. The gap between the pad and guard ring is $50 \mu \mathrm{m}$. At the center of the detector pad, there is a $2 \mathrm{~mm}$ diameter round 
metal opening area allowing for optical excitations. At the periphery of the detector pad (East and West direction) eight round $1 \mathrm{~mm}$ detector pads are located, which are intended for other studies.

The AlN passivation was deposited on both front and back surfaces of the CdTe crystals, hence the $\mathrm{Al}_{2} \mathrm{O}_{3}$ ALD process is by nature a conformal coating. $\mathrm{Al}_{2} \mathrm{O}_{3}$ was deposited at $120^{\circ} \mathrm{C}$ in a Beneq TFS-500 batch-type ALD reactor, using trimethylaluminium (TMA) as the metal precursor and water as the oxidant. AlN was deposited in a MRC-903 sputtering tool using the mixture of Ar $(200 \mathrm{sccm})$ and $\mathrm{N}_{2}(505 \mathrm{sccm})$ gases under the deposition current condition of $10 \mathrm{~A}$. Following dielectric deposition, the contact openings were created by wet etching for both passivation types. The fabrication process sequence is described in reference [25].
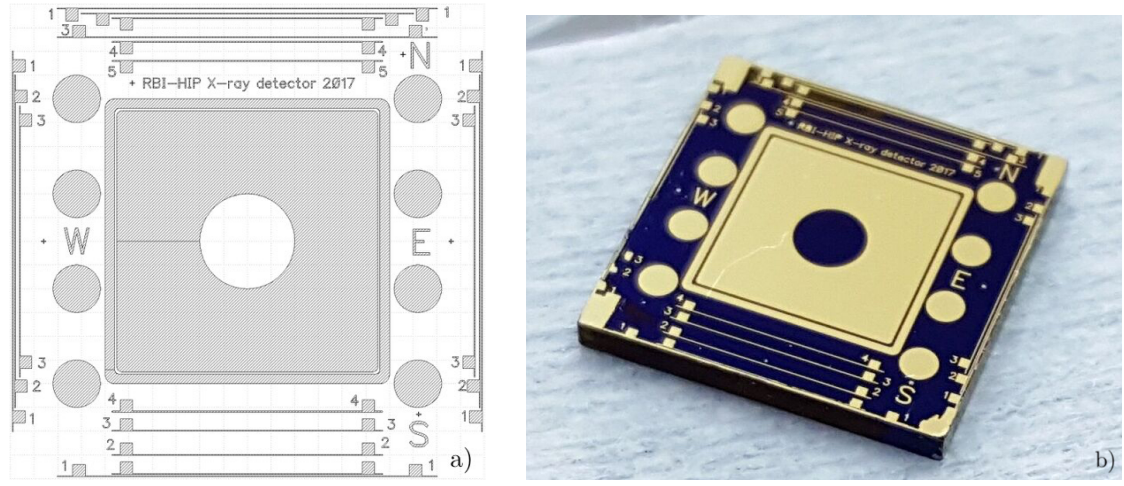

Figure 1: a) Layout of the CdTe pad detector. b) Photograph of a processed detector chip.

\section{Measurements}

\subsection{Transient Current Technique Measurement}

The Transient Current Technique (TCT) is a commonly adopted method to characterize semiconductor detectors. The TCT setup used in this study was constructed by Particulars d.o.o (Ljubljana, Slovenia) [26]. Optical excitation was performed with a red laser $(\lambda=660 \mathrm{~nm})$ directed on the sensor front plane. The illumination generates a cloud of charge carriers within less than $1 \mu \mathrm{m}$ depth from the detector surface. One type of charge carriers, either electrons or holes depending on the device structure, drifts only a few micrometers and is gathered to the electrode so quickly that the resulting signal is damped by the rise-time of the data acquisition electronics. Carriers of the other type drift through the entire thickness of the device resulting in transient current signal, which is detected by an oscilloscope. 
In this case, the $\mathrm{CdTe}$ detectors were biased with positive high voltage from the back plane, so the TCT signal displays electrons drifting through the device, while the holes are immediately collected away at the front contact. This allowed us to study the electron dominated signal formation, which would be the preferred operation mode for segmented CdTe detectors as described above.

In addition to the red laser, the other components in the measurement setup were focusing optics, a sample holder mounted on a XYZ stage for scanning the entire surface of the detector, a $2 \mathrm{kV}$ Bias-T (model BT-01), a wide band current amplifier (model AM-02) all by Particulars d.o.o., a Keithley 24101100 V Source Meter unit, a Tenma power supply, a LeCroy WaveRunner 8404M-MS $4 \mathrm{GHz}$ oscilloscope and a PC and DAQ with MATLAB [27] based software. The laser pulse was transmitted to the detector by an optical fiber. A probehead needle was placed on the active area and a $\mathrm{Cu}$ plate connected the bias circuit to the front and back surfaces, respectively. The laser illumination was directed to the $50 \mu \mathrm{m}$ gap between the pad and guard ring.

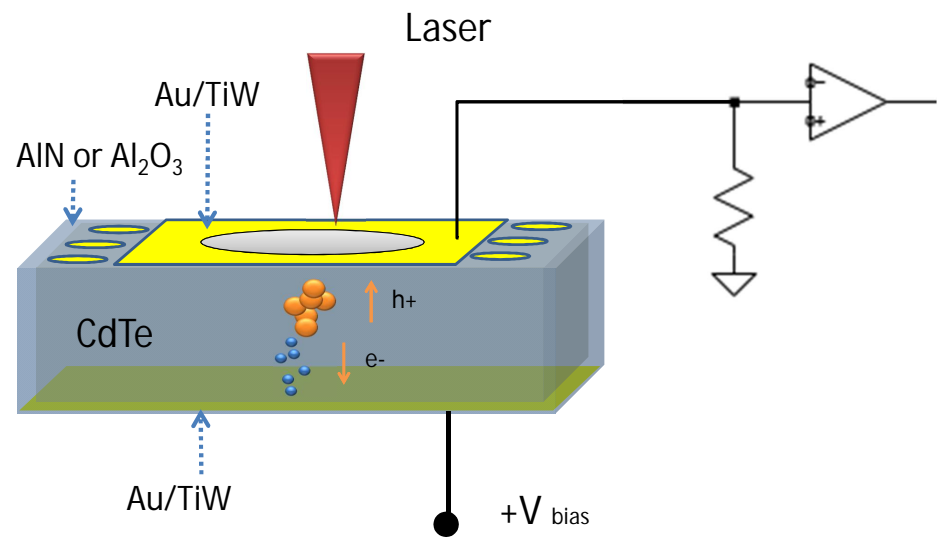

Figure 2: Schematic cross section view of the detector package.

The repetition rate of the laser is adjustable from $5 \mathrm{kHz}$ to $500 \mathrm{kHz}$. During the measurements, more than one hundred waveforms were recorded both for the $\mathrm{Al}_{2} \mathrm{O}_{3}$ and the AlN passivated detectors. An illustrative presentation of the results is shown in Figures 3a and 3b. 

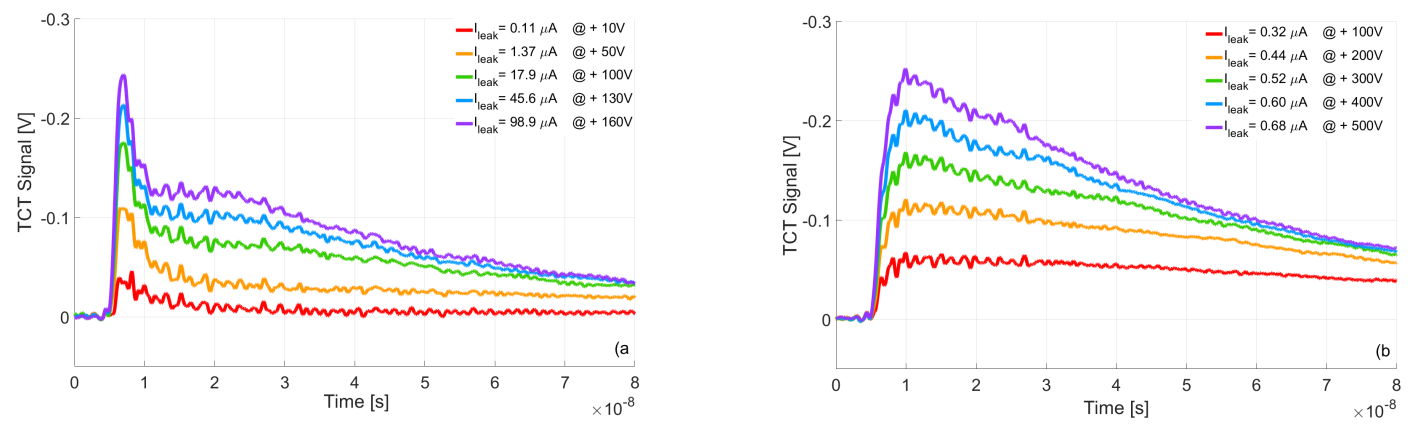

Figure 3: Current transients of a) AlN and b) $\mathrm{Al}_{2} \mathrm{O}_{3}$ passivated CdTe detectors at different bias voltages. (note: vertical scales are arbitrary units.)

The current reading of the sourcing power supply was recorded during the measurements. As it can be seen in Figures 3a and 3b, the AlN-passivated detector could be biased only up to $160 \mathrm{~V}$. The $\mathrm{Al}_{2} \mathrm{O}_{3}$ passivated detector, on the other hand, could be biased up to $500 \mathrm{~V}$, while the current remained about three orders of magnitude smaller than for the AlN sample. Another notable feature when comparing these two detectors is the different pulse shapes. The AlN sample shows rather quick drop of the pulse amplitude, while the signals recorded from $\mathrm{Al}_{2} \mathrm{O}_{3}$ sample remain more flat over longer period of time indicating potentially longer carrier lifetimes. In both cases, the pulse duration is in the order of $80 \mathrm{~ns}$ as expected to be seen from $1 \mathrm{~mm}$ thick detectors.

Long signal decay times are known to be a problem in detectors made of highly defected materials such as CdTe [28, 29]. The polarization in CdTe was studied by continuously illuminating the samples with different red laser pulse repetition rates for up to half an hour. An example of results is shown in Figures $4 \mathrm{a}$ and $4 \mathrm{~b}$.
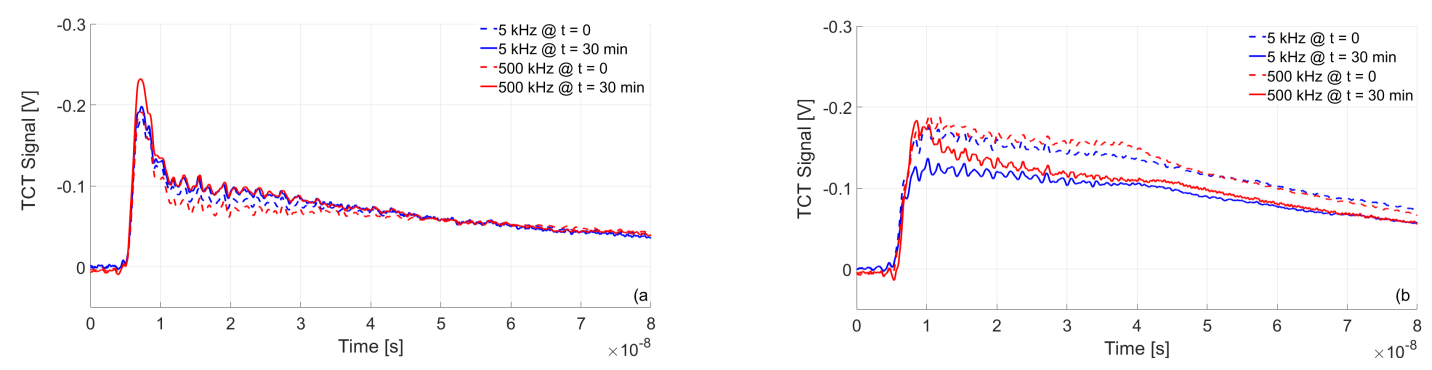

Figure 4: Time evolution of current transients recorded at $5 \mathrm{kHz}$ and $500 \mathrm{kHz}$ laser repetition rates from a) AlN and b) $\mathrm{Al}_{2} \mathrm{O}_{3}$ passivated detector.(note: vertical scales are arbitrary units.) 
It was observed that the signal (integral of current over the time) did not essentially change in the AlN-passivated sample (Figures 4a). In the $\mathrm{Al}_{2} \mathrm{O}_{3}$ sample (Figures $4 \mathrm{~b}$ ) providing generally stronger TCT signals, a decay in the order of about $20 \%$ could be observed. The signal decay was notably independent of optical excitation level from 5 to $500 \mathrm{kHz}$ in range.

\subsection{Simulations}

In order to understand the experimental results, numerical simulations were performed using Synopsys Sentaurus Technology Computer-Aided Design (TCAD) [30] software. The simulated diode structure had dimensions $(100 \times 1000 \times 1) \mu^{3}$ with a $50 \mathrm{~nm}$ thick $\mathrm{AlN}$, or $\mathrm{Al}_{2} \mathrm{O}_{3}$ passivation layer. The material for the contacts on the front and backplanes was Ti. In the simulation, an n-type doped CdTe bulk with a uniform constant doping concentration of $1 \times 10^{11} \mathrm{~cm}^{-3}$ was considered. The diode was biased from the backplane contact. To reproduce the highly defected bulk of the diode, two mid-gap levels (a deep acceptor and a donor level) were implemented with energies $0.58 \mathrm{eV}$ and $0.48 \mathrm{eV}$ and concentration $1 \times 10^{12} \mathrm{~cm}^{-3}$ and $1 \times 10^{15} \mathrm{~cm}^{-3}$, respectively, and electron and hole capture cross sections $1 \times 10^{-13} \mathrm{~cm}^{2}$ and $1 \times 10^{-14} \mathrm{~cm}^{2}$. Furthermore, an additional interface trap at the $\mathrm{CdTe} / \mathrm{Al}_{2} \mathrm{O}_{3}$ interface was added as an acceptor level with the density of $1 \times 10^{12} \mathrm{~cm}^{-3}$.

AlN deposition at low temperatures, such as $150{ }^{\circ} \mathrm{C}$ without a post heat treatment results in a formation of positive fixed oxide charge, $Q_{f}$, at the interface [31]. Whereas, $\mathrm{Al}_{2} \mathrm{O}_{3}$ has a negative fixed oxide charge. Therefore, a positive and negative fixed oxide charges were used for $\mathrm{AlN}$ and $\mathrm{Al}_{2} \mathrm{O}_{3}$, respectively, with the absolute value of $Q_{f}$ equal to $1 \times 10^{12} \mathrm{~cm}^{-3}$ each.

The TCT simulations were carried out using optical excitation induced by a laser with $660 \mathrm{~nm}$ wavelength and a Gaussian-shaped $1 \mathrm{~ns}$ pulse with sigma of $50 \mathrm{ps}$. The illumination was applied next to the front collecting contact. For the generationrecombination mechanism in the CdTe, the doping-dependent Shockley-Reed-Hall model (Scharfetter relation [30]) and impact ionization (van Overstraeten-de Man model [32]) were used. Figure 5 shows a simulated current transients of AlN a) and $\mathrm{Al}_{2} \mathrm{O}_{3}$ b) passivated CdTe detectors. In simulations, $150 \mathrm{~V}$ bias voltage is assumed in both cases. 

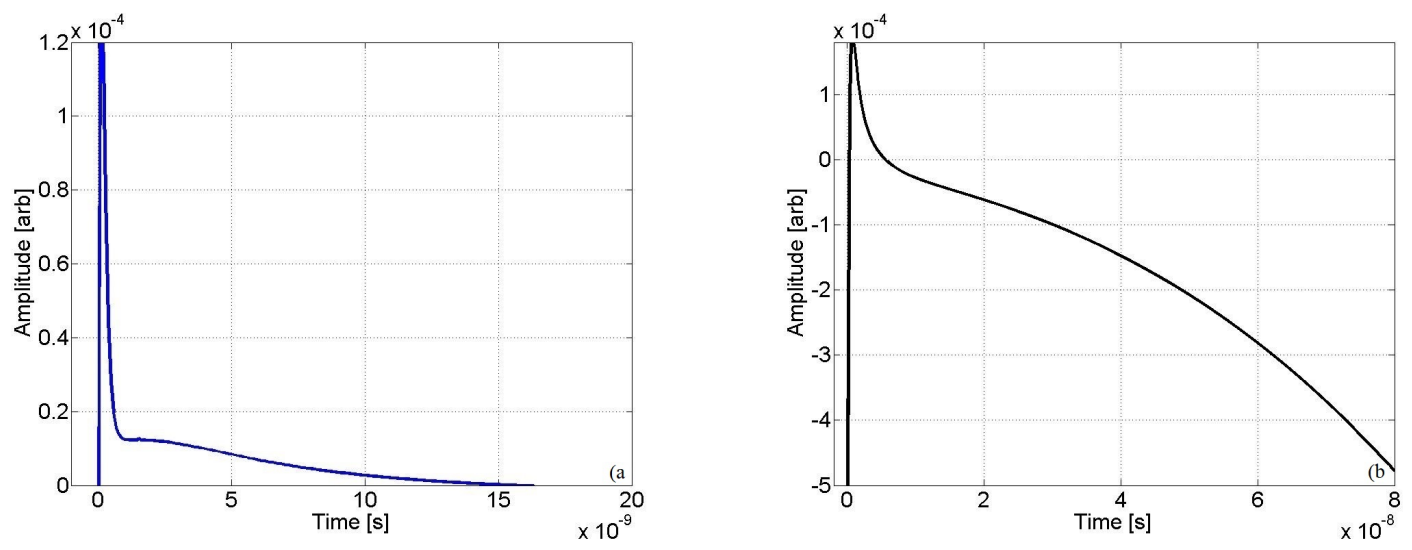

Figure 5: Simulated current transients of a) $\mathrm{AlN}$ and b) $\mathrm{Al}_{2} \mathrm{O}_{3}$ passivated CdTe detectors.

One can observe a sharply rising signal in the first part of transients. Unlike in simulations, which are based on ideal models, this part of transient is filtered out from experimental data since signal rise time is limited by time constants of electrical measurement circuit. Similar pulse shapes as shown Figures 3a and 3b are, however, reproduced by numerical calculations, with the $\mathrm{Al}_{2} \mathrm{O}_{3}$ sample exhibiting a longer pulse duration and a larger signal than the AlN-passivated detector. The electron current transient results in a negative polarity signal, while hole current transient has a positive polarity. In the case of Fig. 5b, it can be seen that for the first few nanoseconds the signal consists of the hole carriers and as time progress it diminishes and electron current prevails. On the material level, transient pulse duration depends on various parameters, e.g. charge carrier lifetime, mobility, and inhomogeneities in bulk and interfaces. Therefore, we account the difference in transient time between simulation and experimental data towards the crystallographic imperfections that were not modeled, as well as the limited time constants of the read out circuit. On the other hand, the fast decreasing signal in case of AlN-passivated sensor is due to changes in electric field induced by positive fixed oxide charge.

\section{Conclusions and summary}

We produced $\mathrm{CdTe}$ detectors with different passivation layers: aluminum oxide $\left(\mathrm{Al}_{2} \mathrm{O}_{3}\right)$ grown by Atomic Layer Deposition (ALD) method and sputtered aluminum nitride $(\mathrm{AlN})$. The CCE of the detectors was studied by recording $660 \mathrm{~nm}$ wavelength laser induced current transients with a TCT measurement setup. During the 
measurement campaign more than one hundred waveforms were recorded in different areas of $\mathrm{Al}_{2} \mathrm{O}_{3}$ and $\mathrm{AlN}$ samples.

Our results systematically indicate longer pulse duration, i.e. higher CCE and longer charge carrier lifetime in $\mathrm{Al}_{2} \mathrm{O}_{3}$-passivated detectors than in ones passivated by the AlN. This is likely due to the negative oxide charge in ALD grown $\mathrm{Al}_{2} \mathrm{O}_{3}$ thin films. The negative charge repulses drifting electrons to be trapped/recombined at the heavily defected surfaces and thus resulting in higher CCE. This experimental observation is supported by numerical TCAD calculations, which reveal longer pulse duration if the oxide charge polarity of a field insulator is swapped from positive to negative and no change in polarization effect of CdTe was observed. These results coincide with our earlier studies made on CdTe pixel detectors fabricated by the same $\mathrm{TiW} / \mathrm{CdTe} / \mathrm{TiW}$ and $\mathrm{Al}_{2} \mathrm{O}_{3}$ passivation methodology. As reported in [25], we detected at room temperature a photopeak at $662 \mathrm{keV}$ with about $2 \%$ energy resolution. Additionally, we observed about $20 \%$ relative signal decay when $\mathrm{CdTe}$ detectors were illuminated at different laser repetition rates, i.e. different charge injection levels, for up to half an hour. While the laser repetition rate has influence onto the TCT signal amplitude, hence the amount of induced and trapped charges, no significant differences were observed for the polarization effect of the CdTe for either of the passivation methods. An operation of 30 minutes is a substantially longer time than what for instance patients would be exposed to by typical medical $\mathrm{X}$-ray imaging devices. Thus, we are confident that $\mathrm{Al}_{2} \mathrm{O}_{3}$ grown at $120^{\circ} \mathrm{C}$ by ALD is a suitable method to provide electrical passivation for CdTe X-ray detectors.

\section{Acknowledgements}

This study has partially been funded by the Horizon 2020 ERA Chair project, grant agreement 669014 and by Academy of Finland project number 314473, "Multispectral photon-counting for medical imaging and beam characterization". J. Ott acknowledges funding from the Viljo, Yrjö and Kalle Väisälä Foundation of the Finnish Academy of Science and Letters. Detector fabrication was performed in the cleanroom facilities of Micronova Nanofabrication Centre in Espoo, Finland. The authors are grateful to Dr. Eija Tuominen, coordinator of the Detector Laboratory at Helsinki Institute of Physics at the University of Helsinki Department of Physics, for providing the environment for electrical measurements.

\section{References}

[1] G. Knoll, Radiation detection and measurement, 4th Edition, John Wiley, Hoboken, N.J, 2010. 
[2] W. A. Kalender, D. Kolditz, C. Steiding, V. Ruth, F. Lück, A.-C. Rößler, E. Wenkel, Technical feasibility proof for high-resolution low-dose photoncounting CT of the breast, European Radiology (2016) 1-6.

[3] P. Simon R Cherry, J. A. Sorenson, M. E. Phelps, Physics in Nuclear Medicine - (Fourth Edition) - ScienceDirect, sciencedirect.com, 2012.

[4] A. Winkler, H. Koivunoro, V. Reijonen, I. Auterinen, S. Savolainen, Prompt gamma and neutron detection in BNCT utilizing a CdTe detector, Applied Radiation and Isotopes 106 (2015) 139-144. doi:10.1016/j.apradiso.2015.07.040.

[5] S. Del Sordo, L. Abbene, E. Caroli, A. M. Mancini, A. Zappettini, P. Ubertini, Progress in the Development of CdTe and CdZnTe Semiconductor Radiation Detectors for Astrophysical and Medical Applications, Sensors 9 (2009) 34913526 .

[6] A. G. Kozorezov, J. K. Wigmore, A. Owens, R. den Hartog, A. Peacock, H. A AlJawhari, Resolution degradation of semiconductor detectors due to carrier trapping, Nuclear Inst. and Methods in Physics Research, A 546 (1-2) (2005) 209212. doi:10.1016/j.nima.2005.03.026.

[7] K. Hecht, Zum Mechanismus des lichtelektrischen Primärstromes in isolierenden Kristallen, Zeitschrift für Physik 77 (3-4) (1932) 235-245.

[8] S. M. Sze, K. K. Ng, Physics of Semiconductor Devices, Wiley, 2006.

[9] S. Csaba, CdZnTe and CdTe materials for X-ray and gamma ray radiation detector applications, physica status solidi (b) 241 (3) (2004) 783-790. doi: $10.1002 /$ pssb.200304296.

[10] A. E. Bolotnikov, N. Abdul-Jabber, S. Babalola, G. S. Camarda, Y. Cui, A. Hossain, E. Jackson, H. Jackson, J. James, K. T. Kohman, A. Luryi, R. B. James, Effects of Te inclusions on the performance of CdZnTe radiation detectors, in: 2007 IEEE Nuclear Science Symposium Conference Record, IEEE, 2009, pp. $1788-1797$.

[11] A. Cola, I. Farella, Electric field and current transport mechanisms in schottky cdte x-ray detectors under perturbing optical radiation, Sensors 13 (7) (2013) 9414-9434. doi:10.3390/s130709414.

URL http://www.mdpi.com/1424-8220/13/7/9414 
[12] K. Suzuki, S. Seto, T. Sawada, K. Imai, Carrier transport properties of HPB CdZnTe and THM CdTe:Cl, IEEE Transactions on Nuclear Science 49 (2002) 1287-1291. doi:10.1109/TNS.2002.1039653.

[13] T. Takahashi, S. Watanabe, Recent progress in CdTe and CdZnTe detectors, IEEE Transactions on Nuclear Science 48 (4) (2001) 950-959.

[14] R. H. Redus, J. A. Pantazis, T. J. Pantazis, A. C. Huber, B. J. Cross, Characterization of CdTe Detectors for Quantitative X-ray Spectroscopy, IEEE Transactions on Nuclear Science.

[15] H. Heikkinen, A. Gädda, S. Vähänen, J. Salonen, P. Monnoyer, G. Blaj, L. Tlustos, M. Campbell, Low-temperature bump bonding of timepix readout chips and cdte sensors at different sensor pitches, in: 2011 IEEE Nuclear Science Symposium Conference Record, 2011, pp. 4770-4775. doi:10.1109/NSSMIC.2011.6154712.

[16] R. N. Jacobs, J. Markunas, J. Pellegrino, L. A. Almeida, M. Groenert, M. Jaime-Vasquez, N. Mahadik, C. Andrews, S. B. Qadri, Role of thermal expansion matching in CdTe heteroepitaxy on highly latticemismatched substrates, Journal of Crystal Growth 310 (12) (2008) 2960-2965. doi:10.1016/j.jcrysgro.2008.02.029.

[17] S. Sze, Semiconductor Devices: Physics and Technology, John Wiley \& Sons Singapore Pte. Limited, 2012.

[18] T. Suntola, Atomic layer epitaxy, Materials Science Reports 4 (5) (1989) 261312 .

[19] M. Leskelä, M. Ritala, Atomic layer deposition (ALD): from precursors to thin film structures, Thin Solid Films 409 (1) (2002) 138-146. doi:10.1016/S00406090(02)00117-7.

[20] J. M. Sturm, A. I. Zinine, H. Wormeester, B. Poelsema, R. G. Bankras, J. Holleman, J. Schmitz, Imaging of oxide charges and contact potential difference fluctuations in atomic layer deposited $\mathrm{Al} 2 \mathrm{O} 3$ on $\mathrm{Si}$, Journal of Applied Physics 97 (6) (2005) 063709. doi:10.1063/1.1870113.

[21] P. Repo, H. Talvitie, S. Li, J. Skarp, H. Savin, Silicon Surface Passivation by Al2O3: Effect of ALD Reactants, Energy Procedia 8 (2011) 681-687. doi:10.1016/j.egypro.2011.06.201. 
[22] J. Härkönen, E. Tuovinen, P. Luukka, A. Gädda, T. Maenpaa, E. Tuominen, T. Arsenovich, A. Junkes, X. Wu, Z. Li, Processing of $n+/ p-/ p+$ strip detectors with atomic layer deposition (ALD) grown $\mathrm{Al} 2 \mathrm{O} 3$ field insulator on magnetic Czochralski silicon (MCz-si) substrates, Nuclear Instruments and Methods in Physics Research Section A: Accelerators, Spectrometers, Detectors and Associated Equipment 828 (2016) 46-51. doi:10.1016/j.nima.2016.04.069.

[23] J. Härkönen, J. Ott, M. Mäkelä, T. Arsenovich, A. Gädda, T. Peltola, E. Tuovinen, P. Luukka, E. Tuominen, A. Junkes, J. Niinistö, M. Ritala, Atomic Layer Deposition (ALD) grown thin films for ultra-fine pitch pixel detectors, Nuclear Inst. and Methods in Physics Research, A 831 (2016) 2-6. doi:10.1016/j.nima.2016.03.037.

[24] Acrorad Ltd., Acrorad webpage, https://www.acrorad.co.jp (accessed on January, 2018).

[25] A. Gädda, A. Winkler, J. Ott, J. Härkönen, A. Karadzhinova-Ferrer, A. Koponen, P. Luukka, J. Tikkanen, S. Vähänen, Advanced processing of cdte pixel radiation detectors, Journal of Instrumentation 12. doi:10.1088/17480221/12/12/C12031.

[26] Particulars, advanced measurement systems, http://particulars.si/index.php (accessed on January, 2018).

[27] Mathworks Inc., Matlat webpage, https://www.mathworks.com (accessed on January, 2018).

[28] A. Cola, I. Farella, The polarization mechanism in CdTe Schottky detectors, Applied Physics Letters 94 (10) (2009) 102113. doi:10.1063/1.3099051.

[29] B. Dezillie, V. Eremin, Z. Li, E. Verbitskaya, Polarization of silicon detectors by minimum ionizing particles, Nuclear Inst. and Methods in Physics Research, A 452 (3) (2000) 440-453. doi:10.1016/S0168-9002(00)00450-2.

[30] Synopsys, Synopsys webpage, https://www.synopsys.com (accessed on January, 2018).

[31] P. Repo, Reducing surface recombination in black silicon photovoltaic devices using atomic layer deposition, Ph.D. thesis, Aalto University, Helsinki, Finland (2016).

URL http://urn.fi/URN : ISBN : 978-952-60-6919-7 
312 [32] R. Van Overstraeten, H. De Man, Measurement of the ionization rates in diffused silicon p-n junctions, Solid-State Electronics 13 (5) (1970) 583-608. 